\title{
An application of the Hooley-Huxley contour
}

\author{
by \\ R. Balasubramanian (Madras), A. Ivić (Beograd) \\ and K. Ramachandra (Bombay)
}

To the memory of Professor Helmut Hasse (1898-1979)

1. Introduction and statement of results. This paper is a continuation of our paper [1]. We begin by stating a special case of what we prove in the present paper.

THEOREM 1. Let $k$ be any complex constant and $(\zeta(s))^{k}=\sum_{n=1}^{\infty} d_{k}(n) n^{-s}$ in $\sigma \geq 2$. Then

$$
\begin{aligned}
& \int_{1}^{T}\left|(\zeta(1+i t))^{k}\right|^{2} d t=T \sum_{n=1}^{\infty}\left|d_{k}(n)\right|^{2} n^{-2}+O\left((\log T)^{\left|k^{2}\right|}\right), \\
& \int_{1}^{T}\left|\frac{\zeta^{\prime}(1+i t)}{\zeta(1+i t)}\right|^{2} d t=T \sum_{m \geq 1} \sum_{p}(\log p)^{2} p^{-2 m}+O\left((\log T)^{2}\right),
\end{aligned}
$$

and

$$
\int_{1}^{T}|\log \zeta(1+i t)|^{2} d t=T \sum_{m \geq 1} \sum_{p}\left(m p^{m}\right)^{-2}+O(\log \log T) .
$$

Remark 1. In [1] we proved (1) with $k=1$ and studied the error term in great detail.

Remark 2. The proof of this theorem and Theorem 3 to follow require the use of the Hooley-Huxley contour as modified by K. Ramachandra in [2] (for some explanations see [3]). We write $m(H H)$ for this contour.

Re mark 3 . We have an analogue of these results for $\zeta$ and $L$-functions of algebraic number fields. In fact, under somewhat general conditions on 
$F(s)=\sum_{n=1}^{\infty} a_{n} n^{-s}$ (or even $\sum_{n=1}^{\infty} a_{n} \lambda_{n}^{-s}$ and so on) we can show that

(4) $\int_{1}^{T}|F(1+i t)|^{2} d t=T \sum_{n=1}^{\infty}\left|a_{n}\right|^{2} n^{-2}+O\left(\log \log T+\sum_{n \leq T^{C}}\left|a_{n}\right|^{2} n^{-1}\right)$

where $C(>0)$ is a large constant.

The following theorem is fairly simple to prove.

TheOREM 2. Let $1=\lambda_{1}<\lambda_{2}<\ldots$ be a sequence of real numbers with $C_{0}^{-1} \leq \lambda_{n+1}-\lambda_{n} \leq C_{0}$ where $C_{0}(\geq 1)$ is a constant and let $a_{1}, a_{2}, \ldots$ be any sequence of complex numbers satisfying the following conditions:

(i) $\sum_{n \leq x}\left|a_{n}\right| n^{-1}=O_{\varepsilon}\left(x^{\varepsilon}\right)$ for all $\varepsilon>0$ and $x \geq 1$.

(ii) $\sum_{n=1}^{\infty}\left|a_{n}\right|^{2} n^{\lambda-2}$ converges for some constant $\lambda$ with $0<\lambda<1$.

(iii) $F(s)=\sum_{n=1}^{\infty} a_{n} \lambda_{n}^{-s}$ (which converges in $\sigma>1$ ) is continuable analytically in $\left(\sigma \geq 1-\delta, t \geq t_{0}\right)$ and there $|F(s)|<t^{A}$, where $\delta(0<\delta<1 / 10)$, $t_{0}(\geq 100)$ and $A(\geq 2)$ are any constants.

Then

$$
\begin{aligned}
\int_{t_{0}+C_{1} \log \log T}^{T} \mid & |F(1+i t)|^{2} d t \\
& =T \sum_{n=1}^{\infty}\left|a_{n}\right|^{2} \lambda_{n}^{-2}+O\left(\log \log T+\sum_{n \leq T^{C_{2}}}\left|a_{n}\right|^{2} n^{-1}\right)
\end{aligned}
$$

where $C_{1}$ and $C_{2}$ are certain positive constants depending on other constants which occur in the definition of $F(s)$.

We sketch a proof of this theorem. We put $s=1+i t, t \geq t_{0}$,

$$
\begin{gathered}
R(w)=\exp \left(\left(\sin \frac{w}{100}\right)^{2}\right), \\
\Delta(u)=\frac{1}{2 \pi i} \int_{2-i \infty}^{2+i \infty} u^{w} R(w) \frac{d w}{w} \quad(u>0),
\end{gathered}
$$

and

(8) $\sum_{n=1}^{\infty} a_{n} \lambda_{n}^{-s} \Delta\left(\frac{X}{\lambda_{n}}\right)=\frac{1}{2 \pi i} \int_{2-i \infty}^{2+i \infty} F(s+w) X^{w} R(w) \frac{d w}{w} \quad\left(X=T^{C_{3}}\right)$,

$C_{3}$ (>0) being a large constant. In the integral just mentioned we cut off the portion $|\operatorname{Im} w| \geq C_{4} \log \log T$ where $C_{4}(>0)$ is a large constant and in the remaining part we move the line of integration to $\operatorname{Re} w=-\delta$. Observe 
that in $|\operatorname{Re} w| \leq 3$ we have

$$
R(w)=O\left(\left(\exp \exp \left(\left|\operatorname{Im} \frac{w}{100}\right|\right)\right)^{-1}\right) .
$$

Without much difficulty we obtain

$$
F(s)=\sum_{n=1}^{\infty} a_{n} \lambda_{n}^{-s} \Delta\left(\frac{X}{\lambda_{n}}\right)+O\left(T^{-2}\right)=A(s)+E(s) \quad \text { say. }
$$

Using a well-known theorem of H. L. Montgomery and R. C. Vaughan we have

$$
\begin{aligned}
\int_{t_{0}+C_{1} \log \log T}^{T} & |A(1+i t)|^{2} d t \\
= & \sum_{n=1}^{\infty}\left|a_{n}\right|^{2} \lambda_{n}^{-2}\left|\Delta\left(\frac{X}{\lambda_{n}}\right)\right|^{2}\left(T-C_{1} \log \log T+O(n)\right) .
\end{aligned}
$$

Now $\Delta(u)=O\left(u^{2}\right)$ always but it is also $1+O\left(u^{-2}\right)$ and using these we are led to the theorem.

However, the proof of Theorem 1 (and also that of Theorem 3) is not simple. It has to use the density results $N(\sigma, T)=O\left(T^{B(1-\sigma)}(\log T)^{B}\right)$ and $N(\sigma, T)=O\left(T^{B^{\prime}(1-\sigma)^{3 / 2}}(\log T)^{B^{\prime}}\right)$ (the former is a consequence of the latter if we are not particular to have a small value of $B)$ where $B(>0)$ and $B^{\prime}(>0)$ are constants and $1-\delta \leq \sigma \leq 1$. Also it has to use the zero free region $\sigma \geq 1-C_{3}(\log t)^{-2 / 3}(\log \log t)^{-1 / 3}\left(t \geq t_{0}\right)$ for the Riemann zeta function (and more general functions). Since the constant $B$ is unimportant in our proof, Remark 3 below Theorem 1 holds. (In fact, as will be clear from our proof, only the portion $\sigma \geq 1-\delta$ of the $m(H H)$ contour will be enough for our purposes.) Also if only the density result $N(\sigma, T)=O\left(T^{B(1-\sigma)}(\log T)^{B}\right)$ and the zero free region $\sigma \geq 1-C_{5}(\log T)^{-1}$ are available then we end up with

$$
O\left(\log \log T+\sum_{n \leq \exp \left((\log T)^{3}\right)}\left|a_{n}\right|^{2} n^{-1}\right)
$$

for the error term and it is not hard to improve this to some extent. We now proceed to state our general result.

Consider the set $S_{1}$ of all abelian $L$-series of all algebraic number fields. We can define $\log L(s, \chi)$ in the half plane $\operatorname{Re} s>1$ by the series

$$
\sum_{m} \sum_{p} \chi\left(p^{m}\right)\left(m p^{m s}\right)^{-1}
$$

where the sum is over all positive integers $m \geq 1$ and $p$ runs over all primes (in the case of algebraic number fields $p$ runs over the norm of all prime ideals). More generally, we can (by analytic continuation) define $\log L(s, \chi)$ 
in any simply connected domain containing $\operatorname{Re} s>1$ which does not contain any zero or pole of $L(s, \chi)$. For any complex constant $z$ we can define $(L(s, \chi))^{z}$ as $\exp (z \log L(s, \chi))$. Let $S_{2}$ consist of the derivatives of $L(s, \chi)$ for all $L$-series and let $S_{3}$ consist of the logarithms as defined above for all $L$-series.

Let $P_{1}(s)$ be any finite power product (with complex exponents) of functions in $S_{1}$. Let $P_{2}(s)$ be any finite power product (with non-negative integral exponents) of functions in $S_{2}$. Also let $P_{3}(s)$ be any finite power product (with non-negative integral exponents) of functions in $S_{3}$. Let $b_{n}(n=1,2,3, \ldots)$ be complex numbers which are $O_{\varepsilon}\left(\exp \left((\log n)^{\varepsilon}\right)\right)$ for every fixed $\varepsilon>0$ and suppose that $F_{0}(s)=\sum_{n=1}^{\infty} b_{n} n^{-s}$ is absolutely convergent in $\operatorname{Re} s \geq 1-\delta$ where $\delta(0<\delta<1 / 10)$ is a positive constant. Finally, put

$$
F(s)=P_{1}(s) P_{2}(s) P_{3}(s) F_{0}(s)=\sum_{n=1}^{\infty} a_{n} n^{-s} .
$$

Then we have

THEOREM 3. We have

$$
\begin{aligned}
& \int_{1}^{T}|F(1+i t)|^{2} d t \\
& \quad=T \sum_{n=1}^{\infty}\left|a_{n}\right|^{2} n^{-2}+O\left(\log \log T+\sum_{n \leq T^{C_{6}}}\left|a_{n}\right|^{2} n^{-1}\right)
\end{aligned}
$$

where $C_{6}(>0)$ is a large constant.

Remark 1 . It is possible to have a more general result. For example we can replace $F(s)$ in (12) and (13) by $F(s)+\sum_{n=1}^{\infty} d_{m}(n)(n+\alpha)^{-s}$ where $m$ is a positive integer constant and $\alpha$ is any constant with $0<\alpha<1$. Then the right hand side of (13) has to be replaced by

$$
\begin{aligned}
T \sum_{n=1}^{\infty}\left|a_{n}\right|^{2} n^{-2}+T \sum_{n=1}^{\infty}\left(d_{m}(n)\right)^{2}( & +\alpha)^{-2}+O(\log \log T) \\
& +O\left(\sum_{n \leq T^{C_{6}}}\left(\left|a_{n}\right|^{2}+\left(d_{m}(n)\right)^{2}\right) n^{-1}\right) .
\end{aligned}
$$

2. Proof of Theorem 3. We form the $m(H H)$ contour (associated with $L$-functions occurring in $F(s))$ as in [2]. But we select a small constant $\delta(0<\delta<1)$ and treat the points $1-\delta+i \nu(\nu=0, \pm 1, \pm 2, \ldots)$ as though they were zeros associated with $L$-functions occurring in $F(s)$. We recall 
$R(w)=\exp \left((\sin (w / 100))^{2}\right)$. Put $s=1+i t, T_{0}=C_{7} \log \log T \leq t \leq T$,

$$
A(s)=\sum_{n=1}^{\infty} a_{n} n^{-s} \Delta\left(\frac{X}{n}\right)
$$

where $\Delta(u)$ and $X$ are as in (8). Then

$$
\frac{1}{2 \pi i} \int_{2-i \infty}^{2+i \infty} F(s+w) X^{w} R(w) \frac{d w}{w}=A(s) .
$$

We write $w=u+i v$ and truncate the portion $|v| \geq \frac{1}{2} T_{0}$ and move the $w$-line of integration so that $s+w$ lies in the portion of the $m(H H)$ contour pertaining to $|v| \leq \frac{1}{2} T_{0}$. We obtain

$$
F(s)=A(s)+E(s)
$$

where for fixed $t$ in $\left(T_{0} \leq t \leq T\right)$,

$$
E(s)=-\frac{1}{2 \pi i} \int_{P} F(s+w) X^{w} R(w) \frac{d w}{w}
$$

where $P$ is the path consisting of the $m(H H)$ contour in $\left(u \geq-\delta,|v| \leq \frac{1}{2} T_{0}\right)$ and the lines connecting it to $\sigma=1$ by lines perpendicular to it at the ends. Notice that to the right of the $m(H H)$ we have (by Lemma 5 of [2])

$$
|F(s+w)| \leq \exp \left((\log t)^{\psi}\right)
$$

with a certain constant $\psi$ (satisfying $0<\psi<1$ ) for $s+w$ on $M_{1,1}$ and $M_{1,2}$ (we adopt the notation of [2]). Also

$$
|F(s+w)| \leq \exp \left((\log T)^{\psi^{\prime}}\right)
$$

with a small constant $\psi^{\prime}\left(0<\psi^{\prime}<1 / 5\right)$ for $s+w$ on $M_{1,3}$. With these we have the following contributions to $\int_{T_{0} / 2}^{T+T_{0} / 2}|E(s)| d t$ and $\int_{T_{0} / 2}^{T+T_{0} / 2}|E(s)|^{2} d t$. We handle the first integral and the treatment of the second is similar. We have (denoting by $P_{1}$ the contour $P$ with the horizontal lines connecting $P$ to $\sigma=1$ omitted)

$$
\begin{aligned}
\int_{T_{0}}^{T}|E(s)| d t & \leq(\log T)^{2} \int_{T_{0}}^{T} \int_{P_{1}}|F(s+w)| X^{u}|d w| d t+T^{-10} \\
& \leq(\log T)^{3} \int_{Q}|F(s)| X^{\sigma-1}|d s|+T^{-10}
\end{aligned}
$$

where $Q$ is the portion of the $m(H H)$ in $\left(\sigma \geq 1-\delta, T_{0} / 2 \leq t \leq T+T_{0} / 2\right)$. (Note that $s$ is used as a variable on the $m(H H)$ in the integral in (20).) 
(In the case of $\int_{T_{0}}^{T}|E(s)|^{2} d t$ we majorise it by

$$
\begin{aligned}
&(\log T)^{4} \int_{T_{0}}^{T}\left(\int_{P_{1}}|F(s+w)| X^{u}|d w|\right)^{2} d t+T^{-10} \\
& \leq(\log T)^{5} \int_{T_{0}}^{T} \int_{P_{1}}|F(s+w)|^{2} X^{2 u}|d w| d t+T^{-10}
\end{aligned}
$$

by Hölder's inequality.)

The contribution to (20) from $M_{1,1}$ is

$$
O\left((\log T)^{20} \max _{1-\delta \leq \sigma \leq 1-\tau_{1}}\left(N(\sigma, T) X^{-(1-\sigma)}\right) \exp \left((\log T)^{\psi}\right)\right)
$$

and that from $M_{1,2}$ is

$$
O\left((\log T)^{20} \max _{1-\tau_{1} \leq \sigma \leq 1-\tau_{2}}\left(N(\sigma, T) X^{-(1-\sigma)}\right) \exp \left((\log T)^{\psi^{\prime}}\right)\right)
$$

and that from $M_{1,3}$ is

$$
O\left((\log T)^{D} \exp \left((\log T)^{\psi^{\prime}}\right) X^{-\tau_{3}}\right)
$$

where $\tau_{1}$ and $\tau_{2}$ are determined by $M_{1,1}, M_{1,2}$ and $M_{1,3}$ and $\tau_{3}=$ $C_{3}(\log T)^{-2 / 3}(\log \log T)^{-1 / 3}$. Here $D(>0)$ is some constant. (Note that $X$ is a large positive constant power of $T$.) Using the standard estimates (for some details which are very much similar to what we need, see equations (1) $-(3)$ of [3]) we obtain

Lemma 1. Both $\int_{T_{0}}^{T}|E(s)| d t$ and $\int_{T_{0}}^{T}|E(s)|^{2} d t$ are $O\left(\exp \left(-(\log T)^{0.1}\right)\right)$.

Lemma 2. We have $A(s)=O\left(\exp \left((\log T)^{\varepsilon}\right)\right)$.

Proof. Follows from the fact that

$$
|A(s)| \leq \sum_{n=1}^{\infty}\left|a_{n}\right| n^{-1}\left|\Delta\left(\frac{X}{n}\right)\right| .
$$

Lemma 3. The integral $\int_{T_{0}}^{T}|A(s) E(s)| d t$ is $O\left(\exp \left(-\frac{1}{2}(\log T)^{0.1}\right)\right)$.

Proof. Follows from Lemmas 1 and 2.

LEMMA 4. We have

$$
\int_{T_{0}}^{T}|F(s)|^{2} d t=\int_{T_{0}}^{T}|A(s)|^{2} d t+O\left(\exp \left(-\frac{1}{2}(\log T)^{0.1}\right)\right) .
$$

Proof. Follows from Lemmas 2 and 3. Now the integral on the right hand side of (21) is

$$
\sum_{n=1}^{\infty}\left(T-T_{0}+O(n)\right)\left|a_{n}\right|^{2} n^{-2}\left|\Delta\left(\frac{X}{n}\right)\right|^{2}
$$


by a well-known theorem of H. L. Montgomery and R. C. Vaughan, and so Theorem 3 follows by a slight further work since $a_{n}=O_{\varepsilon}\left(n^{\varepsilon}\right)$ for all $\varepsilon>0$.

\section{References}

[1] R. Balasubramanian, A. Ivić and K. Ramachandra, The mean square of the Riemann zeta-function on the line $\sigma=1$, Enseign. Math. 38 (1992), 13-25.

[2] K. Ramachandra, Some problems of analytic number theory, I, Acta Arith. 31 (1976), 313-324.

[3] A. Sankaranarayanan and K. Srinivas, On the papers of Ramachandra and Kátai, ibid. 62 (1992), 373-382.

MATSCIENCE

THARAMANI P.O

MADRAS 600 113, INDIA

KATEDRA MATEMATIKE RGF-a

UNIVERSITET u. BEOGRADU, DJUŠINA 7

BEOGRAD, YUGOSLAVIA
SCHOOL OF MATHEMATICS

TATA INSTITUTE OF FUNDAMENTAL RESEARCH

HOMI BHABHA ROAD

BOMBAY 400 005, INDIA 